\title{
Lobar Microbleeds Are Associated with a Decline in Executive Functioning in Older Adults
}

\author{
Irene B. Meier ${ }^{a, b}$ Yian Gu ${ }^{a}$ Vanessa A. Guzaman ${ }^{a}$ Anne F. Wiegman ${ }^{a}$ \\ Nicole Schupf ${ }^{a, c, d}$ Jennifer J. Manly ${ }^{a, c, e}$ José A. Luchsinger ${ }^{d, f}$ \\ Anand Viswanathang Sergi Martinez-Ramirez ${ }^{g}$ Steven M. Greenberg ${ }^{g}$ \\ Richard Mayeux ${ }^{\text {a,c-e }}$ Adam M. Brickman a, c, e

\begin{abstract}
aTaub Institute for Research on Alzheimer's Disease and the Aging Brain, College of Physicians and Surgeons, Columbia University, New York, N.Y., USA; b Division of Psychiatry Research and Psychogeriatric Medicine, University of Zurich, Zurich, Switzerland; ${ }^{\circ}$ G.H. Sergievsky Center, College of Physicians and Surgeons, Columbia University, New York, N.Y., d Department of Epidemiology, Mailman School of Public Health, Columbia University, New York, N.Y., e'Department of Neurology, College of Physicians and Surgeons, Columbia University, New York,

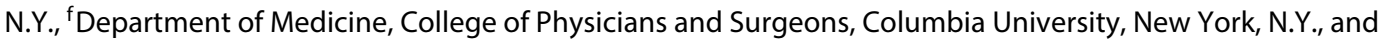 \\ gDepartment of Neurology, Massachusetts General Hospital, Harvard Medical School, Boston, Mass., USA
}

\section{Key Words}

Microbleeds · Cognition · Cerebral amyloid angiopathy · $\mathrm{MRI} \cdot$ Longitudinal design

\footnotetext{
Abstract

Background: Normal aging is associated with a decline in cognitive abilities, particularly in the domains of psychomotor speed and executive functioning. However, 'aging,' per se, is not a cause of cognitive decline but rather a variable that likely captures multiple accumulating biological changes over time that collectively affect mental abilities. Recent work has focused on the role of cerebrovascular disease as one of the biological changes. In the current study, we examined whether lobar microbleeds - magnetic resonance imaging (MRI) signal voids due to hemosiderin deposits secondary to cerebral amyloid angiopathy - are associated with cognitive decline in normal aging. Previous studies that reported a relationship between the presence of lobar micro-
}

bleeds and decreased cognitive abilities have been primarily cross-sectional. Here, we used a retrospective longitudinal design to examine whether the presence of lobar microbleeds is associated with the rate of cognitive decline among non-demented older adults. Methods: Participants came from an ongoing longitudinal community-based aging study, in which subjects are evaluated at 18-24 months intervals and received a full medical, neurological, and neuropsychological examination at each of the follow-up visits. Gradient echo MRI scans were available on 197 non-demented participants (mean age: $84.15 \pm 5.02$ years). Microbleeds were rated visually on axial view and divided into subcortical (basal ganglia, cerebellum) and lobar (frontal, temporal, parietal, occipital lobe) regions, and confirmed with coronal and sagittal views to exclude artifacts. Cognition was assessed with a neuropsychological battery, providing summary scores for memory, language, executive, and visuospatial abilities. Using general estimating equations (GEE), we compared cognition cross-sectionally between individuals

\section{KARGER}

E-Mail karger@karger.com www.karger.com/ced (c) 2014 S. Karger AG, Basel

$1015-9770 / 14 / 0385-0377 \$ 39.50 / 0$
Adam M. Brickman

Taub Institute for Research on Alzheimer's Disease and the Aging Brain Department of Neurology College of Physicians and Surgeons, Columbia University 630 West 168th Street, P\&S Box 16, New York, NY 10032 (USA)

E-Mail amb2139@columbia.edu 
with 2 or more $(n=11)$ and fewer than $2(n=186)$ lobar microbleeds and examined longitudinal cognitive change beginning $9.47 \pm 3.13$ years before the MRI scan. Results: Subjects with 2 or more lobar microbleeds had worse executive functioning at the visit closest to the MRI scan ( $\beta=-0.044$; $p<0.001)$ and had a faster decline in executive function over time ( $\beta=-0.072 ; p=0.012$ ) than subjects with fewer than 2 lobar microbleeds. The two groups were similar in age at scan date, education, ethnicity, sex distribution, and cognitive performance at first visit. Conclusions: Lobar microbleeds, a marker of cerebral amyloid angiopathy, are associated with an accelerated rate of executive function decline. The presence of cerebral amyloid angiopathy may be an important source of cognitive decline in aging. Future work should examine how cerebral amyloid angiopathy interacts with neurodegenerative processes, such as Alzheimer's disease.

(c) 2014 S. Karger AG, Basel

\section{Introduction}

Over the past several decades, there have been myriad efforts to characterize the nature of age-associated cognitive decline. Most studies agree that aging is associated with a gradual loss of cognitive abilities, with increasing variability or individual differences [1-3] particularly in the domains of psychomotor speed and executive function [4]. 'Aging', per se, is not a cause of cognitive decline but rather a variable that likely captures multiple accumulating biological changes over time that collectively affect mental abilities.

There are currently no accepted definitions of what constitutes 'normal' versus 'pathological' cognitive aging. Typically, we consider normal cognitive aging as a decline in certain cognitive abilities in the absence of frank disease or evidence of neurodegenerative pathology [5]. Several authors have argued that small vessel cerebrovascular disease is a primary source of normal cognitive aging [68]. 'Pathological cognitive aging', on the other hand typically refers to cognitive decline that is due to a neurodegenerative condition [9]. In the case of Alzheimer's disease $(\mathrm{AD})$, the most common cause of pathological aging, current hypothetical pathogenic models emphasize the precipitating role of fibrillar forms of beta amyloid protein and tau pathology, which ultimately cause neurodegeneration and the neuropsychological syndrome associated with the disease [10]. The amyloid cascade hypothesis suggests that imbalance of production and clearance of $A \beta$ is a primary driver of pathological cognitive aging.
Recent literature, however, implicates small vessel cerebrovascular disease as an important source of dysfunction in $\mathrm{AD}$ and perhaps disease pathogenesis [8], suggesting some overlap between factors that promote cognitive decline due to normal aging and due to AD. Cerebral amyloid angiopathy (CAA), or the deposition of beta amyloid in blood vessel walls, may represent a mechanistic link between amyloid-centered hypothesis and the increasing attention toward vascular factors in AD pathogenesis - promoting cognitive decline in individuals without dementia but also contributing to the pathogenesis of AD. Cerebral amyloid angiopathy manifests radiologically as cerebral microbleeds (MB) distributed in lobar regions on gradient echo (GRE) and susceptibility weighted (SWI) magnetic resonance imaging (MRI) $[11,12]$. Thus, lobar microbleeds are considered a radiological indicator of the presence of underlying CAA, and associations with clinical characteristics involving microbleeds can be attributed to the role of the underlying amyloid pathology.

Previous studies showed that lobar microbleeds are associated with reduced cognition cross-sectionally [1315 ], and that individuals with $\mathrm{AD}$ have a greater number of lobar microbleeds [16] and CAA [17] compared with healthy individuals. However, efforts to examine longitudinal cognitive change as a function of microbleed status are scarce and have only been carried out in clinical populations [18]. There are no studies to our knowledge that have examined the association of CAA, appreciated radiologically as lobar microbleeds, and longitudinal cognitive change among non-demented older adults. In the current study, we used a retrospective longitudinal design to examine whether community-dwelling individuals with 2 or more lobar microbleeds, as an indicator of CAA, had a more precipitous rate of cognitive change than individuals with fewer than 2 lobar microbleeds.

\section{Materials and Methods}

\section{Participants}

Subjects were participants in an ongoing longitudinal community-based study of aging and dementia in northern Manhattan, which had two recruitment waves beginning in 1992 and 1999. Participants were all English or Spanish speaking, over age 65, and either White, Hispanic, or African American. Recruitment procedures and sampling strategies have been described in detail previously [19]. Participants were evaluated at 18-24 month intervals and they received a full medical, neurological, and neuropsychological examination at each of the follow-up visits. A history of hypertension was ascertained by self-report [20]. Beginning in 2005 , participants not meeting criteria for dementia at their previous follow-up visit were invited to participate in an MRI study [21]. 
Seven hundred sixty-nine participants underwent high-resolution initial MRI.

Beginning in 2009, participants with initial MRI scans were invited for a follow-up MRI scan, leading to a total of 339 subjects with repeat MRI; 243 of the 339 had T2*-weighted gradient echo (GRE) scans for microbleed assessment [22]. For the current analysis, we examined cognition data starting in 1999 and excluded subjects meeting clinical criteria for dementia at the time of the GRE MRI scan $(\mathrm{n}=46)$, resulting in a sample of 197 subjects (mean age: $84.15 \pm 5.02$ years) that comprised the study group reported here. The average time between the baseline cognitive assessment and the subjects' GRE MRI scan was $9.47(\mathrm{SD}=3.13)$ years, with an average of 4.7 visits per subject (range: 3-11). The GRE MRI scan was conducted at the visit closest to the last neuropsychological examination included in the current study.

\section{MRI Protocol}

High-resolution, three-dimensional T2*-weighted GRE images $(\mathrm{TR}=45 \mathrm{~ms}, \mathrm{TE}=31 \mathrm{~ms}$, flip angle $=13$, slice thickness $=2 \mathrm{~mm}$, in plane resolution $1 \times 1 \mathrm{~mm}$ ) were acquired on a Philips Intera 1.5 Tesla MRI scanner (Best, the Netherlands) for microbleed quantification. Microbleeds were rated by visual inspection following the criteria put forth by the Microbleed Study Group [11]: dark, round lesions on GRE images within the parenchyma and at least half way surrounded by a white rim. They were rated on axial slices, and confirmed by visual inspection of corresponding sagittal and coronal planes to exclude artifacts such as calcium deposits, partial volume artifacts, cavernous malformations, bone, or vessel flow voids. Microbleeds were divided into 'lobar' (frontal, temporal, parietal, occipital lobes) or 'deep' (basal ganglia, thalamus, and cerebellum) categories. Number and location of microbleeds were recorded for each subject. All microbleeds were evaluated by a single blinded operator (IBM) after establishing excellent reliability with a 'gold standard' rater (SMR; intra-class correlation for reliability analysis of 20 images $=0.94)$.

We divided our sample into two groups: subjects with zero or one $(<2)$ lobar microbleeds and subjects with two or more $(2+)$ lobar microbleeds, indicating evidence of CAA. By focusing on individuals with 2 or more lobar microbleeds, we increased our confidence that the experimental group had evidence of CAA because multiple lobar lesions are a strong indicator of underlying pathology and a single lesion is not uncommon among individuals without pathological evidence of CAA [23].

As previously described [22], total white matter hyperintensities (WMH) volumes were calculated for concurrent MRI scans and the presence of radiological infarcts was evaluated at the subjects' baseline MRI scan.

\section{Neuropsychological Evaluation}

Cognitive performance was assessed with a comprehensive neuropsychological battery, and summary scores for the domains memory, language, speed/executive functioning, and visuospatial abilities were derived. Summary scores were calculated through exploratory and confirmatory factor analyses and represented on a $\mathrm{z}$-distribution [24].

\section{Statistical Analyses}

We compared the demographic characteristics at the time of scan and the first cognitive test performance between individuals with fewer than two $(<2)(\mathrm{n}=186)$ and individuals with two or more $(2+)$ lobar microbleeds $(\mathrm{n}=11)$. A retrospective longitudinal design was used to examine the rates of cognitive decline preceding the MRI scan, beginning $9.47( \pm 3.13)$ years prior to the scan, as a function of the microbleed group, using general estimating equations (GEE) [25]. The primary effects of interest in the GEE model include Group, Time, and the Group $\times$ Time interaction. A main effect of Time would indicate a significant change in cognitive abilities over time. A significant Group effect would indicate that subjects with two or more lobar microbleeds differ from subjects with fewer than two lobar microbleeds at the time of first visit (intercept). A significant Group $\times$ Time interaction would indicate that the rate of cognitive change over time differs between the two microbleeds groups. We ran separate analyses for summary measures of memory, language, speed/executive functioning, and visuospatial abilities. The variables age at MRI scan, years of education, ethnicity, sex, and cognitive domain scores at baseline were entered into the GEE model as covariates.

\section{Results}

Individuals in the two groups were similar in age at scan date, education, ethnicity, sex distribution, frequency of hypertension, and cognitive performance at the time of the first visit (table 1). The prevalence of microbleeds in the entire sample was $27.2 \%$. The 11 subjects with $2+$ lobar microbleeds had a mean number of 4.45 microbleeds ( \pm 3.205$)$. In the $<2$ microbleeds group, 30 subjects had one lobar microbleed and 156 subjects had no microbleeds. Six subjects had both deep and lobar microbleeds. We re-ran the analyses excluding these subjects and the reported effects did not change notably. Individuals with $2+$ lobar microbleeds had greater total WMH volumes and were more likely to have a radiological infarct than individuals with $<2$ lobar microbleeds.

Table 2 displays the results of the GEE analysis. For speed/executive functioning, both groups declined over time (significant main effect of Time) but participants with $2+$ lobar microbleeds declined at a faster rate than those with $<2$ lobar microbleeds (significant Group $\times$ Time interaction; see fig. 1). The two groups had similar speed/executive functioning performance at their first assessment (nonsignificant main effect of Group). Of the covariates in the model, age, education, baseline cognition, but not sex were associated with speed/executive functioning.

For the other cognitive domains, the rate of cognitive decline was similar between the two microbleeds groups (nonsignificant Group $\times$ Time effects). Like for speed/ executive functioning, both groups declined in memory and language, but not visuospatial functioning over time (significant main effects of Time). The two microbleeds 
Table 1. Subject demographic data

\begin{tabular}{|c|c|c|c|c|}
\hline Age at date of MRI scan & $84.14 \pm 5.06$ & $84.39 \pm 4.44$ & $84.15 \pm 5.02$ & $\mathrm{t}=0.619 ; \mathrm{p}=0.871$ \\
\hline Education, years (mean \pm SD) & $11.84 \pm 4.65$ & $10.00 \pm 6.73$ & $11.75 \pm 4.78$ & $\mathrm{t}=1.190 ; \mathrm{p}=0.235$ \\
\hline Speed/executive function at first visit (mean $\pm \mathrm{SD})$ & $0.48 \pm 0.06$ & $0.19 \pm 0.51$ & $0.46 \pm 0.92$ & $\mathrm{t}=0.965 ; \mathrm{p}=0.336$ \\
\hline Visuospatial functioning at first visit (mean $\pm \mathrm{SD})$ & $0.49 \pm 0.51$ & $0.38 \pm 0.66$ & $0.48 \pm 0.52$ & $\mathrm{t}=0.699 ; \mathrm{p}=0.485$ \\
\hline Sex (\% women) & 68.2 & 60.0 & 67.52 & $\mathrm{X}^{2}=0.517 ; \mathrm{p}=0.350$ \\
\hline Ethnicity (\% African American/Hispanic/White) & $34 / 27 / 39$ & $46 / 33 / 21$ & $35 / 29 / 36$ & $\mathrm{X}^{2}=1.992 ; \mathrm{p}=0.369$ \\
\hline Hypertension, $\mathrm{n}(\%)$ & $124(66 \%)$ & $10(91 \%)$ & $134(68 \%)$ & $\mathrm{X}^{2}=2.81 ; \mathrm{p}=0.09$ \\
\hline WMH volume, $\log$ transformed, $\mathrm{cm}^{3}($ mean $\pm \mathrm{SD})$ & $1.46 \pm 1.30$ & $2.31 \pm 1.02$ & $1.48 \pm 1.48$ & $\mathrm{t}=2.40 ; \mathrm{p}=0.039$ \\
\hline Radiological infarct, $\mathrm{n}(\%)$ & $50(27 \%)$ & $6(55 \%)$ & $56(28 \%)$ & $\mathrm{X}^{2}=3.85 ; \mathrm{p}=0.05$ \\
\hline
\end{tabular}

Table 2. GEE analyses for the domains speed, memory, language, and visuospatial functioning

\begin{tabular}{|c|c|c|c|c|c|c|c|c|}
\hline \multirow[t]{2}{*}{ Variable } & \multicolumn{2}{|c|}{$\begin{array}{l}\text { Speed/executive } \\
\text { function }\end{array}$} & \multicolumn{2}{|c|}{ Memory } & \multicolumn{2}{|c|}{ Language } & \multicolumn{2}{|c|}{ Visuospatial } \\
\hline & $\beta$ & $\mathrm{p}$ & $\beta$ & $\mathrm{p}$ & $\beta$ & $\mathrm{p}$ & $\beta$ & $\mathrm{p}$ \\
\hline $\begin{array}{c}\text { Group }(0=<2 \text { microbleeds } \\
1=2+\text { microbleeds })\end{array}$ & -0.392 & 0.108 & 0.122 & 0.307 & -0.055 & 0.660 & 0.001 & 0.857 \\
\hline Time & -0.044 & $<0.001$ & -0.028 & $<0.001$ & -0.010 & 0.003 & -0.061 & 0.699 \\
\hline Group $\times$ time & -0.072 & 0.012 & 0.016 & 0.288 & -0.005 & 0.793 & 0.026 & 0.137 \\
\hline Age & -0.013 & 0.013 & -0.014 & 0.002 & -0.010 & 0.001 & -0.007 & 0.009 \\
\hline $\operatorname{Sex}(0=M, 1=F)$ & -0.006 & 0.912 & 0.097 & 0.050 & 0.047 & 0.113 & 0.057 & 0.039 \\
\hline Education & 0.017 & 0.019 & 0.015 & 0.024 & 0.014 & 0.019 & 0.021 & $<0.001$ \\
\hline Baseline cognition & 0.774 & $<0.001$ & 0.726 & $<0.001$ & 0.804 & $<0.001$ & 0.686 & $<0.001$ \\
\hline
\end{tabular}

Beta values are standardized.

Fig. 1. Differential decline in speed/executive functioning among individuals with 2 or more microbleeds versus controls. Plotted results of GEE model examining the Group $\times$ Time interaction for the cognitive domain of speed/executive function, adjusted for age, education, ethnicity, sex, and cognitive performance at first visit. The MRI scan for microbleed assessment was completed at year 0 .

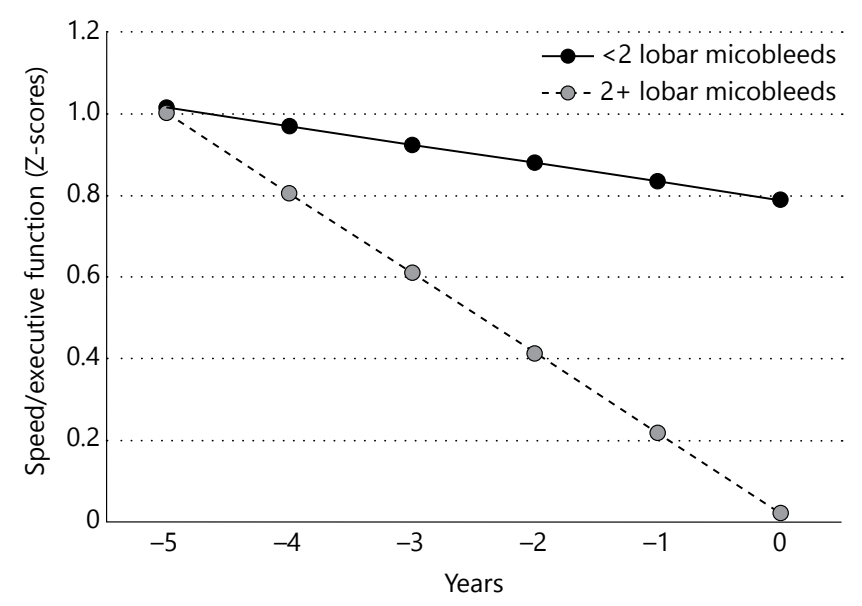

Meier et al. 
groups were similar in their abilities at baseline (non-significant main effects of Group). Age, education, and baseline cognitive performance were associated with memory, language, and visuospatial functioning (see table 2). Sex was related only to visuospatial functioning.

\section{Discussion}

We used a retrospective longitudinal design to assess the relationship between lobar microbleeds, as a marker of cerebral amyloid angiopathy, and cognitive decline. Individuals with 2 or more lobar microbleeds had an accelerated rate of speed/executive function decline over the 9-year period preceding the MRI scan relative to individuals with fewer than 2 microbleeds. Unsurprisingly and as we have reported previously [22], individuals with more than 2 microbleeds also had increased evidence of ischemic cerebrovascular disease (i.e., WMH and infarct).

Our findings of reduced cognition in the presence of lobar microbleeds are consistent with previous reports: lobar microbleeds have been associated with decreased overall cognitive status, as well as lower cognitive abilities in specific domains such as psychomotor speed and executive function [14, 26-28]. Werring and colleagues [13] found that $60 \%$ of individuals with lobar microbleeds had executive dysfunction compared with $30 \%$ without microbleeds. Lobar microbleeds also predicted executive functioning impairment 6 years later in a sample of stroke patients [18]. Furthermore, lobar microbleeds are associated with executive impairment in patients with ischemic stroke and transient ischemic attack [29].

Our study extends these findings by examining the longitudinal cognitive course of neurologically healthy individuals with and without evidence of cerebral amyloid angiopathy. Although the retrospective design precludes the ability to determine whether the development of the lobar microbleeds preceded the cognitive outcomes, the findings do establish a relationship between the two. That is, we are unable to conclude definitively whether the microbleeds potentiated more rapid cognitive decline or whether the cognitive decline preceded the microbleeds. The second possibility would suggest that additional factors promote the more rapid cognitive decline and also lead to the development of lobar microbleeds. Prospective analyses will help us determine whether lobar microbleeds promote subsequent cognitive decline in this cohort.

We observed an association of lobar microbleeds with a decline in speed/executive functioning but not in other cognitive domains. Speed or executive tasks may be most sensitive to vascular pathology or subtle age-associated brain changes in general [30]. Further, accelerated decline in executive functioning may be a harbinger for future decline in cognition related to $\mathrm{AD}$. It is noteworthy that among cognitively normal adults above age 60, fibrillar forms of beta amyloid, as measured by positron emissions tomography, was related to reductions in measures of processing speed, working memory, and reasoning, but not declarative memory, a hallmark feature of frank $\mathrm{AD}$ [31]. In the current study, we appear to have a similar association between vascular forms of beta amyloid and reduction in speeded tasks and executive abilities in non-demented older adults. Whether vascular and fibrillar forms of beta amyloid reflect the same pathological process and contribute independently to cognitive outcomes remains an important question. The fact that both can be present in non-demented older adults and are related to variability in cognition and cognitive decline provides compelling evidence that differences between 'normal' and 'pathological' cognitive aging may be more a question of degree than of categorical distinction.

Relatively a few participants in the study had evidence of cerebral amyloid angiopathy by virtue of having two or more lobar microbleeds, but this observation is consistent with what has been reported in previous communitybased cohorts [27]. It should be noted that while deep microbleeds appear to reflect primarily injury associated with hypertensive vasculopathy, lobar microbleeds, which are the focus of the current study, primarily reflect CAA but could also be impacted by ischemia [32]. Although reliable changes in cognition were observed among those with two or more lobar microbleeds, we may have had limited power to detect change in other domains. Furthermore, the relatively low field strength of the MRI scanner used may have led to an underestimation of the number of microbleeds and could account for the few subjects with two or more microbleeds. On the other hand, because of the low field strength, we can be confident that individuals displaying 2 or more lobar microbleeds do in fact have some degree of CAA. Furthermore, our sample is typical of the demography that increasingly comprises the older segment of the US population: racially and ethnically diverse individuals with a range of educational background. Previous efforts that examined associations between lobar microbleeds and cognition generally did so among clinical patients with history of stroke (e.g., [18]); our findings show that CAA is a source of cognitive decline even among community- 
dwelling older adults that were recruited with epidemiological methods.

Similarly, the small number of subjects with 2 or more detectable lobar microbleeds coupled with the relatively high number of covariates included in the GEE models may raise some concern about the reliability of the findings. To address this issue, we selected 11 individuals with $<2$ lobar microbleeds matched on cognition at first visit, age, education, and sex and re-analyzed the data without demographic covariates (data not shown). The observed effects in this limited sample were of the same magnitude as those observed in the presented analyses above, increasing confidence in our findings.

We were able to exploit over 9 years of longitudinal data to examine associations between the rates of cognitive decline and evidence of CAA at a single time point. To our knowledge, this is the first study to examine the longitudinal course of cognition as it relates to CAA in community-dwelling older adults. Our observations have significant theoretical and clinical implications. The find- ings highlight that pathological changes in normal aging and AD may be less distinct than previously thought. That is, vascular forms of beta amyloid, one of the primary pathological features of $\mathrm{AD}$, are prevalent among older adults and predict rate of 'normal' cognitive decline. This shared pathology between individuals with and without dementia due to $\mathrm{AD}$ highlights that there are other factors, besides amyloid, that might potentiate the neuropsychological syndrome that leads to dementia. Nonetheless, microbleeds may be a logical clinical treatment or preventive target to consider when designing studies or interventions to maximize cognitive aging in general. Further, clinicians should take into account the potential role of microbleeds in diagnostic and prognostic formulations.

\section{Acknowledgment}

This work was supported by grants from NIH (AG034189, AG037212, AG042483), the VELUX Foundation, and the Synapsis Foundation.

\section{References}

1 Raz N, Lindenberger U, Rodrigue KM, Kennedy KM, Head D, Williamson A, Dahle C, Gerstorf D, Acker JD: Regional brain changes in aging healthy adults: general trends, individual differences and modifiers. Cereb Cortex 2005;15:1676-1689.

$>2$ Hultsch DF, MacDonald SWS: Intraindividual variability in performance as a theoretical window onto cognitive aging. Oxford, UK, Oxford University Press, 2004.

$\checkmark 3$ Schaie KW, Willis SL: Handbook of the psychology of aging, ed 7. Elsevier, Elsevier, 2011.

4 Grieve SM, Williams LM, Paul RH, Clark CR, Gordon E: Cognitive aging, executive function, and fractional anisotropy: a diffusion tensor MR imaging study. AJNR Am J Neuroradiol 2007;28:226-235.

$>5$ Daffner KR: Promoting successful cognitive aging: a comprehensive review. J Alzheimers Dis 2010;19:1101-1122.

6 Ylikoski R, Ylikoski A, Erkinjuntti T, Sulkava R, Raininko R, Tilvis R: White matter changes in healthy elderly persons correlate with attention and speed of mental processing. Arch Neurol 1993;50:818-824.

7 Gunning-Dixon FM, Raz N: The cognitive correlates of white matter abnormalities in normal aging: a quantitative review. Neuropsychology 2000;14:224-232.

$>8$ Pantoni L: Cerebral small vessel disease: from pathogenesis and clinical characteristics to therapeutic challenges. Lancet Neurol 2010;9: 689-701.
19 Yankner BA, Lu T, Loerch P: The aging brain. Annu Rev Pathol 2008;3:41-66.

10 Jack CR Jr, Knopman DS, Jagust WJ, Shaw LM, Aisen PS, Weiner MW, Petersen RC, Trojanowski JQ: Hypothetical model of dynamic biomarkers of the Alzheimer's pathological cascade. Lancet Neurol 2010;9:119128.

11 Greenberg SM, Vernooij MW, Cordonnier C, Viswanathan A, Al-Shahi Salman R, Warach S, Launer LJ, Van Buchem MA, Breteler MM; Microbleed Study Group: Cerebral microbleeds: a guide to detection and interpretation. Lancet Neurol 2009;8:165174.

12 Cordonnier C, van der Flier WM: Brain microbleeds and Alzheimer's disease: innocent observation or key player? Brain 2011;134: 335-344.

$>13$ Werring DJ, Frazer DW, Coward LJ, Losseff NA, Watt H, Cipolotti L, Brown MM, Jager HR: Cognitive dysfunction in patients with cerebral microbleeds on T2*-weighted gradient-echo MRI. Brain 2004;127:22652275

14 Goos JD, Kester MI, Barkhof F, Klein M, Blankenstein MA, Scheltens P, van der Flier WM: Patients with Alzheimer disease with multiple microbleeds: relation with cerebrospinal fluid biomarkers and cognition. Stroke 2009;40:3455-3460.

15 Poels MM, Ikram MA, van der Lugt A, Hofman A, Niessen WJ, Krestin GP, Breteler MM, Vernooij MW: Cerebral microbleeds are associated with worse cognitive function: the Rotterdam Scan Study. Neurology 2012;78: 326-333.

16 Pettersen JA, Sathiyamoorthy G, Gao FQ, Szilagyi G, Nadkarni NK, St George-Hyslop P, Rogaeva E, Black SE: Microbleed topography, leukoaraiosis, and cognition in probable Alzheimer disease from the Sunnybrook dementia study. Arch Neurol 2008;65:790795.

17 Nakata-Kudo Y, Mizuno T, Yamada K, Shiga K, Yoshikawa K, Mori S, Nishimura T, Nakajima K, Nakagawa M: Microbleeds in Alzheimer disease are more related to cerebral amyloid angiopathy than cerebrovascular disease. Dement Geriatr Cogn Disord 2006;22: 8-14.

18 Gregoire SM, Smith K, Jager HR, Benjamin M, Kallis C, Brown MM, Cipolotti L, Werring DJ: Cerebral microbleeds and long-term cognitive outcome: longitudinal cohort study of stroke clinic patients. Cerebrovasc Dis 2012; 33:430-435.

19 Tang MX, Cross P, Andrews H, Jacobs DM, Small S, Bell K, Merchant C, Lantigua R, Costa R, Stern Y, Mayeux R: Incidence of AD in African-Americans, Caribbean Hispanics, and Caucasians in northern Manhattan. Neurology 2001;56:49-56.

20 Luchsinger JA, Reitz C, Honig LS, Tang MX, Shea S, Mayeux R: Aggregation of vascular risk factors and risk of incident Alzheimer disease. Neurology 2005;65:545551 . 
21 Brickman AM, Schupf N, Manly JJ, Luchsinger JA, Andrews H, Tang MX, Reitz C, Small SA, Mayeux R, DeCarli C, Brown TR: Brain morphology in older African Americans, Caribbean Hispanics, and Whites from northern Manhattan. Arch Neurol 2008;65:1053-1061.

-22 Wiegman AF, Meier IB, Schupf N, Manly JJ, Guzman VA, Narkhede A, Stern Y, MartinezRamirez S, Viswanathan A, Luchsinger JA, Greenberg SM, Mayeux R, Brickman AM: Cerebral microbleeds in a multiethnic elderly community: demographic and clinical correlates. J Neurol Sci 2014;345:125-130.

23 De Reuck J, Deramecourt V, Cordonnier C, Leys D, Maurage CA, Pasquier F: The impact of cerebral amyloid angiopathy on the occurrence of cerebrovascular lesions in demented patients with Alzheimer features: a neuropathological study. Eur J Neurol 2011;18:913-918.

-24 Siedlecki KL, Manly JJ, Brickman AM, Schupf N, Tang MX, Stern Y: Do neuropsychological tests have the same meaning in Spanish speakers as they do in English speakers? Neuropsychology 2010;24:402-411.

25 Zeger SL, Liang KY: Longitudinal data analysis for discrete and continuous outcomes. Biometrics 1986;42:121-130.
26 Poels MM, Ikram MA, Vernooij MW, Krestin GP, Hofman A, Niessen WJ, van der Lugt A, Breteler MM: Total cerebral blood flow in relation to cognitive function: the Rotterdam Scan Study. J Cereb Blood Flow Metab 2008; 28:1652-1655.

27 van Es AC, van der Grond J, de Craen AJ, Westendorp RG, Bollen EL, Blauw GJ, Greenberg SM, van Buchem MA; PROSPER Study Group: Cerebral microbleeds and cognitive functioning in the PROSPER study. Neurology 2011;77:1446-1452.

28 Yakushiji Y, Nishiyama M, Yakushiji S, Hirotsu T, Uchino A, Nakajima J, Eriguchi M, Nanri Y, Hara M, Horikawa E, Kuroda Y: Brain microbleeds and global cognitive function in adults without neurological disorder. Stroke 2008;39:3323-3328.
29 Gregoire SM, Scheffler G, Jaeger HR, Yousry TA, Brown MM, Kallis C, Cipolotti L, Werring DJ: Strictly lobar microbleeds are associated with executive impairment in patients with ischemic stroke or transient ischemic attack. Stroke 2013;44:1267-1272.

30 Nyenhuis DL, Gorelick PB, Geenen EJ, Smith CA, Gencheva E, Freels S, deToledo-Morrell $\mathrm{L}$ : The pattern of neuropsychological deficits in Vascular Cognitive Impairment-No Dementia (Vascular CIND). Clin Neuropsychol 2004; 18:41-49.

31 Rodrigue KM, Kennedy KM, Devous MD Sr, Rieck JR, Hebrank AC, Diaz-Arrastia R, Mathews D, Park DC: $\beta$-Amyloid burden in healthy aging: regional distribution and cognitive consequences. Neurology 2012;78:387395.

-32 Park JH, Seo SW, Kim C, Kim GH, Noh HJ, Kim ST, Kwak KC, Yoon U, Lee JM, Lee JW, Shin JS, Kim CH, Noh Y, Cho H, Kim HJ, Yoon CW, Oh SJ, Kim JS, Choe YS, Lee KH, Lee JH, Ewers M, Weiner MW, Werring DJ, Na DL: Pathogenesis of cerebral microbleeds: in vivo imaging of amyloid and subcortical ischemic small vessel disease in 226 individuals with cognitive impairment. Ann Neurol 2013;73:584-593. 